\title{
The weak cosmic censorship conjecture and thermodynamics in the quintessence AdS black hole under charge particle absorption
}

\author{
Ke-Jian $\mathrm{He}()^{1}$, Xin-Yun $\mathrm{Hu}()^{2,1)}$, Xiao-Xiong Zeng ()$^{3,4}$ \\ 1 Physics and Space College, China West Normal University, Nanchong 637000, China \\ ${ }^{2}$ College of Economic and Management, Chongqing Jiaotong University, Chongqing 400074, China \\ ${ }^{3}$ Department of Mechanics, Chongqing Jiaotong University, Chongqing 400074, China \\ ${ }^{4}$ State Key Laboratory of Mountain Bridge and Tunnel Engineering, Chongqing Jiaotong University, \\ Chongqing 400074, China
}

\begin{abstract}
Considering the cosmological constant as the pressure, we mainly study the laws of thermodynamics and weak cosmic censorship conjecture in the Reissner-Nordström-AdS black hole surrounded by quintessence dark energy under charged particle absorbtion. The first law of thermodynamics is found to be valid as a particle is absorbed by the black hole. The second law however is found to be violated for the extremal and near-extremal black holes since the entropy of these black hole decrease. Moreover, we find that the extremal black hole do not change it configuration in the extended phase space, implying that the weak cosmic censorship conjecture is valid. Remarkably, the near-extremal black hole can be overcharged beyond the extremal condition under charged particle absorption. That is, the cosmic censorship conjecture could be violated for the near-extremal black hole in the extended phase space. To make a comparison, we also discuss the first law, second law as well as the weak cosmic censorship conjecture in the normal phase space, and find that all of them are valid in this case.
\end{abstract}

Keywords: thermodynamics; the weak cosmic censorship conjecture ; the quintessence AdS black hole.

PACS: $04.20 . \mathrm{Dw}, 04.70 . \mathrm{Dy}, 04.20 . \mathrm{Bw}$

\section{Introduction}

Black holes are very important not only in astronomy but also in gravitation theory. For a black hole, there is a geometric singularity located at its center. If the event horizon of the black hole is unstable, the singularity of the black hole will be exposed. The exposed singularity will send uncertain information, leading to the broken of the causal relation between space and time. To avoid this situation, Penrose conjectured that the singularity should be wrapped in the center of the event horizon of the black hole, which is the so-called weak cosmic censorship conjecture [1, 2]. This conjecture also illuminates that the gravitational collapse of dust will eventually form a black hole with singularity [2], which was proved by Penrose and Hawking [3].

Since there is no general method to prove the weak cosmic censorship conjecture in black holes, we need to check its validity in various spacetimes. One interesting method is to check whether the final states of black holes are still black holes after the absorption. In the four-dimensional space time, Wald proved that particles making an extremal Kerr-Newman black hole overcharge or overspin would not be absorbed through Gedanken experiment [4. This result is also applicable to scalar field [5, 6. Later, Hubeny found that for a near-extremal Reissner-Nordström black hole, the weak cosmic censorship conjecture was violated for the charge can exceed its extremum boundary [7]. This behavior was also observed in the near-extremal Kerr and Kerr-Newman black hole [8, 9 . However, as the self-force effect was considered in the Kerr-Newman black hole, the weak cosmic censorship conjecture was found to be still valid for the event horizon was stable 10 12. In addition, the near-extremal Reissner-Nordström black hole are also valid for this conjecture with consideration of the back-reaction effect [13, 14. Many studies have been made on the validity of the weak cosmic censorship conjecture under different black hole backgrounds [15] 40, such as black holes in Einstein's gravity theory, modified gravitational theory, black holes with low or high dimensions, and black holes with electric charge.

Received

1) E-mail: huxinyun@126.com

(c)2019 Chinese Physical Society and the Institute of High Energy Physics of the Chinese Academy of Sciences and the Institute of Modern Physics of the Chinese Academy of Sciences and IOP Publishing Ltd 
The cosmological constant is fixed initially. But with some later studies, the cosmological constant was considered as a variable quantity [41, 42 and the extended phase space is constructed, where the pressure of the black hole is related to the cosmological constant [43, 44, and its thermal conjugate is the thermodynamic volume of the black hole [45, 46]. In this case, the mass of the black hole corresponds to the enthalpy, not the internal energy of the black hole thermodynamic system [47, 48].

In the extended phase space, the first law of thermodynamics has been investigated extensively. However, there is little work to investigate the second law and the weak cosmic censorship conjecture. The validity of the first law dose not mean the second law as well as the weak cosmic censorship conjecture are valid, it thus important and necessary to study the second law and the weak cosmic censorship in the extended phase space. Recently, Ref. [49] investigated the first law, second law, as well as the weak cosmic censorship conjecture in the Reissner Nordström-AdS black holes. It was found that the first law and the weak cosmic censorship conjecture were valid, the second law was violated for the extremal and near-extremal black holes. In this study, we will extend the idea in Ref. [9] to the black holes with quintessence dark energy. We want to explore whether the second law is valid. As a result, we find that the second law is violated too for the extremal and near-extremal black holes. In other hand, it a note that we investigate the change of the minimum value of the metric function at the higher order in the process of particle absorption. This is different from their research. In Ref. [49, they think that $\mathcal{O}\left(\epsilon^{2}\right)$ is the high order terms of $\epsilon$, so it can be considered to zero. However, we can not easily ignore the value of the high order term due to we can not determine the value magnitude of the $\delta_{\epsilon}$ and $\mathcal{O}\left(\epsilon^{2}\right)$. Hence, we have extended the investigate of the changes of the minimum value of the function to the higher order. Moreover, our results show that the weak cosmic censorship conjecture could be violated for the near-extremal black hole in the extended phase space. This result has not appeared in previous studies.

The remainder of this article is organized as follows. In section 2, we briefly review the thermodynamics of the Reissner-Nordström-AdS black hole surrounded by quintessence dark energy. In section 3, we establish the first law of thermodynamics in the extended phase space firstly, and then we discuss the second law and weak cosmic censorship conjecture in this framework. In section 4, we investigate the first law, second law as well as the weak cosmic censorship conjecture in the normal phase space. Section 5 is devoted to our conclusions.

\section{Review of the Reissner-Nordström-AdS black hole surrounded by quintessence dark energy}

The metric of the spherically symmetric charged-AdS black hole surrounded by quintessence dark energy can be written as [50]

$$
d s^{2}=-f(r) d t^{2}+f^{-1}(r) d r^{2}+r^{2}\left(d \theta^{2}+\sin ^{2} \theta d \phi^{2}\right),
$$

with

$$
f(r)=1-\frac{a}{r^{3 \omega+1}}+\frac{r^{2}}{l^{2}}-\frac{2 M}{r}+\frac{Q^{2}}{r^{2}}
$$

the electric potential of the black hole is

$$
A_{\mu}=\left(-\frac{Q}{r}, 0,0,0\right)
$$

In the above equation, $M$ and $Q$ are the ADM mass and charge of the black hole, $l$ is the radius of the $A d S$ spacetime which is related to the cosmological constant. And $a$ is the normalization factor closely related to quintessence density and should be greater than zero. There are several circumstances for the value of $\omega$, for $-1<\omega<-1 / 3$, it is quintessence dark energy, and for $\omega<-1$, it is phantom dark energy. The values of state parameter $\omega$ affect the structure of spacetime. When $\omega=-1$, it affects the AdS radius, while when $\omega=-1 / 3$, it affects the curvature $\kappa$ of space-time [51].

At the event horizon $r_{h}$, the Hawking-temperature, Bekenstein-Hawking entropy and electric potential can be expressed as

$$
\begin{gathered}
T_{h}=\frac{f^{\prime}\left(r_{h}\right)}{4 \pi}=\frac{r_{h}\left(3 a \omega \mathrm{r}_{h}{ }^{-3 \omega}+3 r_{h}{ }^{3} / l^{2}+r_{h}\right)-Q^{2}}{4 \pi \mathrm{r}_{h}{ }^{3}}, \\
S_{h}=\pi r_{h}^{2},
\end{gathered}
$$




$$
\Phi_{h}=-A_{t}\left(r_{h}\right)=\frac{Q}{r_{h}}
$$

In the extended thermodynamic phase space, the cosmological constant play the role of pressure $P$ [43, 44, and its thermal conjugate variable is the thermodynamic volume $V$ of the black hole [45, 46]. The pressure and volume can be expressed as

$$
P=-\frac{\Lambda}{8 \pi}=\frac{3}{8 \pi l^{2}}, \quad V_{h}=\left(\frac{\partial M}{\partial P}\right)_{S, Q}=\frac{4 \pi r_{h}^{3}}{3} .
$$

The first law of thermodynamics thus should be written as [52, 53 ]

$$
d M=T_{h} d S_{h}+\Phi_{h} d Q+V_{h} d P
$$

In this case, the mass is defined as enthalpy. The relations among the enthalpy, internal energy and pressure are

$$
M=U_{h}+P V_{h}
$$

In the extended phase space, the change of mass will affect not only the event horizon and the electric charge, but also the $A d S$ radius. Therefore, we will investigate the change in the black hole by the charged particle absorption.

\section{Thermodynamic and Weak Cosmic Censorship Conjecture with contributions of pressure and volume}

\subsection{Energy-momentum relation of the absorbed particle}

In order to obtain the relationship between the conserved quantities of particles in the electric field $A_{\mu}$, we will employ the following Hamilton-Jacobi equation to study the dynamical of the particles [50].

$$
g^{\mu \nu}\left(p_{\mu}-\mathrm{eA}_{\mu}\right)\left(p_{\nu}-\mathrm{eA}_{\nu}\right)+\mu_{b}^{2}=0
$$

where

$$
p_{\mu}=\partial_{\mu} S
$$

In the above equation, $\mu_{b}$ is the mass of the particle, $p_{\mu}$ is the momentum, and $S$ is the Hamilton action of the particle. In the spherically symmetric spacetime, the Hamilton action of moving particle can be separated into

$$
S=-E t+R(r)+H(\theta)+L \phi
$$

here $E$ and $L$ are the energy and angular momentum. To solve the Hamilton-Jacobi equation, we will use the inverse metric of the black hole as follows

$$
g^{\mu \nu} \partial_{\mu} \partial_{\nu}=-\frac{1}{f(r)}\left(\partial_{t}\right)^{2}+f(r)\left(\partial_{r}\right)^{2}+\frac{1}{r^{2}}\left(\partial_{\theta}\right)^{2}+\frac{1}{r^{2} \sin ^{2} \theta}\left(\partial_{\phi}\right)^{2} .
$$

So the Hamilton-Jacobi equation changes into

$$
-\frac{1}{f(r)}\left(-E-\mathrm{eA}_{t}\right)^{2}+f(r)\left(\partial_{r} S(r)\right)^{2}+\frac{1}{r^{2}}\left(\partial_{\theta} H(\theta)\right)^{2}+\frac{1}{r^{2} \sin ^{2} \theta} L^{2}+\mu_{b}^{2}=0 .
$$

Substituting equation (12) into equation (14), we can get the radial and angular equations

$$
\begin{gathered}
-\frac{r^{2}}{f(r)}\left(-E-\mathrm{eA}_{t}\right)^{2}+r^{2} f(r)\left(\partial_{r} S(r)\right)^{2}+r^{2} \mu_{b}^{2}=-\mathcal{K} \\
\left(\partial_{\theta} H(\theta)\right)^{2}+\frac{1}{\sin ^{2} \theta} L^{2}=\mathcal{K}
\end{gathered}
$$


Correspondingly, the radial momentum $p^{r}$ and angular momentum $p^{\theta}$ of the particle can be written as

$$
\begin{gathered}
p^{r}=f(r) \sqrt{\frac{-\mu_{b}^{2} r^{2}-\mathcal{K}}{r^{2} f(r)}+\frac{1}{f(r)^{2}}\left(-E-e A_{t}\right)^{2}}, \\
p^{\theta}=\frac{1}{r^{2}} \sqrt{\mathcal{K}-\frac{1}{\sin ^{2} \theta} L^{2}} .
\end{gathered}
$$

When the black hole absorbs a charged particle completely, the conserved quantity of the particle and the conserved quantity of the black hole are indistinguishable by an observer outside the horizon. By removing the separate variable $\mathcal{K}$ in equation (17), we can obtain the relationship between the energy and momentum at any radial location. Near the event horizon, we can get

$$
E=\frac{Q}{r_{h}} e+p^{r}
$$

For the $p^{r}$ term, we will choose the positive sign thereafter as done in 54 in order to assure a positive time direction.

\subsection{Thermodynamics in the extended phase space}

In the process of absorption, the energy and electric charge of the particle equal to the change of the internal energy and charge of the black hole, that is

$$
E=d U_{h}=d\left(M-P V_{h}\right), \quad e=d Q .
$$

In this case, the energy relation of equation $(19)$ becomes as

$$
d U_{h}=\frac{Q}{r_{h}} d Q+p^{r}
$$

In addition, in order to rewrite equation (21) to the first law of thermodynamics, we have to find the variation of the entropy. From equation (5), we have

$$
d S_{h}=2 \pi r_{h} d r_{h},
$$

where $d r_{h}$ is the variation of the event horizon of the black hole. The absorbed particles also change the function $f(r)$, the shift of function $f(r)$ satisfies

$$
d f_{h}=\frac{\partial f_{h}}{\partial M} d M+\frac{\partial f_{h}}{\partial Q} d Q+\frac{\partial f_{h}}{\partial l} d l+\frac{\partial f_{h}}{\partial r_{h}} d r_{h}=0, \quad f_{h}=f\left(M, Q, l, r_{h}\right),
$$

where

$$
\begin{aligned}
& \frac{\partial f_{h}}{\partial M}=-\frac{2}{r_{h}}, \quad \frac{\partial f_{h}}{\partial Q}=\frac{2 Q}{r_{h}{ }^{2}}, \\
& \frac{\partial f_{h}}{\partial l}=-\frac{2 r_{h}{ }^{2}}{l^{3}}, \quad \frac{\partial f_{h}}{\partial r_{h}}=-\frac{2 Q^{2}}{r_{h}{ }^{3}}+\frac{2 M}{r_{h}{ }^{2}}+\frac{2 r_{h}}{l^{2}}-a r_{h}{ }^{-2-3 w}(-1-3 w) .
\end{aligned}
$$

Combining equations (21) and 20, the energy relation in the equation 21) can be rewritten as

$$
d M-d\left(P V_{h}\right)=\frac{Q}{r_{h}} d Q+p^{r} .
$$

Combining equations (23) and (25), we find that all the variables are eliminated except for $d r_{h}$ and $p^{r}$, so we can get

$$
d r_{h}=\frac{2 l^{2} p^{r} r_{h}{ }^{1+3 w}}{r_{h}{ }^{3 w}\left(r_{h}{ }^{3}-2 l^{2}\left(M-r_{h}\right)\right)+a l^{2}(3 w-1)} .
$$


Base on equation (26), we can get the variation of entropy and volume

$$
\begin{aligned}
& d S_{h}=\frac{4 l^{2} \pi p^{r} r_{h}{ }^{2+3 w}}{r_{h}{ }^{3 w}\left(r_{h}{ }^{3}-2 l^{2}\left(M-r_{h}\right)\right)+a l^{2}(3 w-1)}, \\
& d V_{h}=\frac{8 l^{2} \pi p^{r} r_{h}{ }^{3+3 w}}{r_{h}{ }^{3 w}\left(r_{h}{ }^{3}-2 l^{2}\left(M-r_{h}\right)\right)+a l^{2}(3 w-1)} .
\end{aligned}
$$

Incorporating equations (4), (27), (7) and (28), we get lastly

$$
T_{h} d S_{h}-P d V_{h}=p^{r} .
$$

Moreover, incorporating equations (4), (5), (6), (7), (27) and (28), the energy relation of equation (21) can be expressed as

$$
d U_{h}=\Phi_{h} d Q+T_{h} d S_{h}-P d V_{h}
$$

Because the mass of the black hole has been defined as enthalpy, the relation between the internal energy and enthalpy can be rewritten in the extended phase space as

$$
d M=T_{h} d S_{h}+\Phi_{h} d Q+V_{h} d P .
$$

Here, we prove that the first law of thermodynamics is still satisfied for the black hole surrounded by quintessence dark energy under the charged particle absorption.

As the absorption is an irreversible process, the entropy of the final state should be greater than the initial state of the black hole. That is, under the charged particle absorption, the variation of the entropy is $d S>0$. Next, we will test the validity of the second law of thermodynamics with equation (27).

We first study the case of the extremal black holes, for which the temperature is zero. On the basis of this fact and equation 27), we can get

$$
d S_{h}=-\frac{4 \pi p^{r} l^{2}}{3 r_{h}} .
$$

There is a minus sign in equation (32). Therefore, the entropy is decreased for the extremal black hole. That is, the second law of thermodynamics is violated for the extremal black hole in extended phase space. In addition, it is worth noting that the parameters $a$ and $\omega$ are not present in the above equation. In other words, the violations about the second law does not depend on the parameters $a$ and $\omega$.

Now, we focus on the near-extremal black hole. We will check whether the second law is valid in the extended phase space by studying the variation of entropy numerically. We set $M=0.5$ and $l=p^{r}=1$. For the case $\omega=-2 / 3$ and $a=1 / 3$, we find the extremal charge is $Q_{e}=0.48725900857$. In the case that the charge is less than the extremal charge, we take different charge values to produce the variation of entropy. In Table 1, we give the numeric results of $r_{h}$ and $d S$ for different charges. As can be seen from Table 1, when the charge $Q$ of the black hole decreases, the event horizon of the black hole increases, and the variation of entropy increase too. Interestingly, there are two regions where the entropy increase are $d S>0$ and $d S<0$. It means that there exist a phase transition point which divides the variation of entropy into positive value and negative value.

Table 1. The relation between $d S_{h}, Q$ and $r_{h}$.

\begin{tabular}{ccc}
\hline$Q$ & $r_{h}$ & $d S_{h}$ \\
\hline 0.48725900857 & 0.432041 & -9.69538 \\
0.487259 & 0.432111 & -9.70328 \\
0.48 & 0.494855 & -22.9663 \\
0.46 & 0.567768 & -1006.44 \\
0.445 & 0.5757 & 386.566 \\
0.4 & 0.628743 & 44.8667 \\
0.3 & 0.695449 & 24.9494 \\
0.2 & 0.731872 & 21.0262 \\
0.1 & 0.751055 & 19.5985 \\
\hline
\end{tabular}


We also can obtain the relation between $d S$ and $r_{h}$, which is shown in Figure 1. From Figure 1, we can clearly see that there really exist a phase transition point making $d S_{h}$ positive and negative. When the electric charge is close to the extremal charge, the variation of entropy is negative. And when the electric charge is far away from the extremal charge, the entropy increases. Therefore, the second law of thermodynamics is violated for the near-extremal black holes and valid for the far-extremal black holes in the extended phase space.

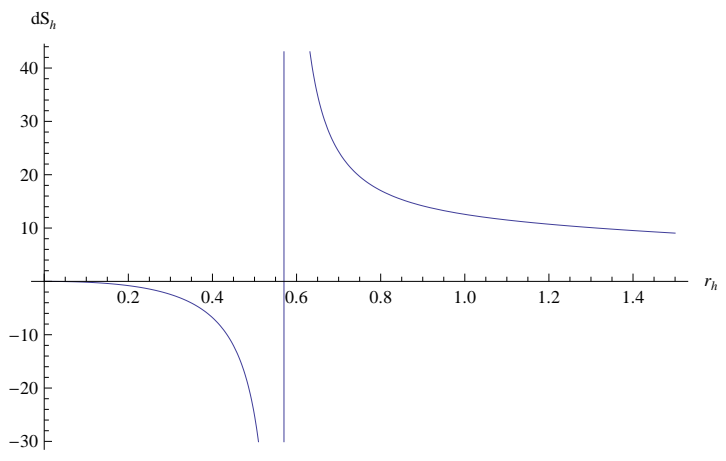

Fig. 1. The relation between $d S_{h}$ and $r_{h}$ which parameter values are $M=0.5, l=p^{r}=1$ and $\omega=-2 / 3, a=1 / 3$.

In Figure 2 and Table 2, we set $\omega=-1 / 2$ and $a=1 / 2$. We want to explore whether the values of state parameter of dark energy affect the laws of thermodynamics. For $\omega=-1 / 2$ and $a=1 / 2$, the extremal charge is $Q_{e}=0.525694072$. Form Figure 2 and Table 2, we also find that the second law of thermodynamics fails for the near-extremal black holes when a particle is absorbed by the black hole. In addition, by comparing Figure 1 and Figure 2, we find that the magnitudes of the violations are related to values of the parameters $\omega$ and $a$, but parameters $\omega$ and $a$ do not determine whether the second law of thermodynamics will eventually be violated.

Table 2. The relation between $d S_{h}, Q$ and $r_{h}$.

\begin{tabular}{ccc}
\hline$Q$ & $r_{h}$ & $d S_{h}$ \\
\hline 0.525694072 & 0.483844 & -8.65919 \\
0.525 & 0.504022 & $-10,2582$ \\
0.5 & 0.599642 & -27.2971 \\
0.43 & 0.69007 & -766.564 \\
0.425 & 0.694382 & 20923.7 \\
0.4 & 0.713732 & 171.512 \\
0.3 & 0.767156 & 50.6936 \\
0.2 & 0.798062 & 37.5343 \\
0.1 & 0.814681 & 33.2405 \\
\hline
\end{tabular}

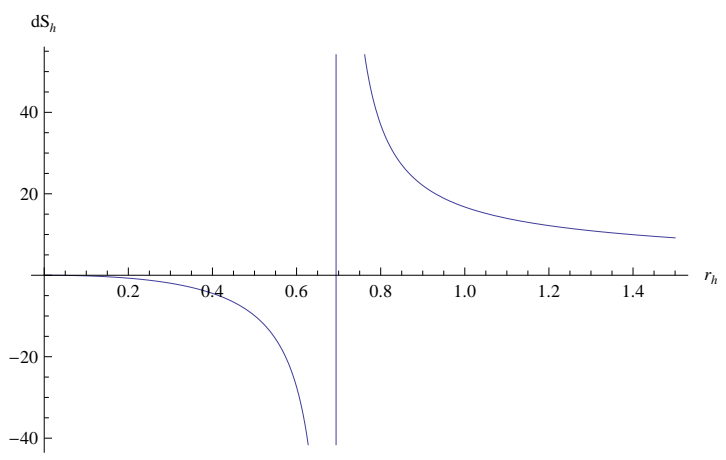

Fig. 2. The relation between $d S_{h}$ and $r_{h}$ which parameter values are $M=0.5, l=p^{r}=1$ and $\omega=-1 / 2, a=1 / 2$. 


\subsection{Weak Cosmic Censorship Conjecture in the extended phase space}

In the extended phase space with consideration of the thermodynamic volume, although the particle absorption is an irreversible process, the second law of thermodynamics for the extremal and near-extremal black holes are violated. As well as known, the definition of entropy is $S_{h}=\frac{A_{h}}{4}$, the event horizon of black holes is closely relevant to entropy. Duo to this violation of the second law of the thermodynamics is found in the case considering thermodynamic volume. It implies that, the violation of the second law of thermodynamics can be related to the weak cosmic censorship conjecture which is related to the stability of the event horizon. Therefore, it is necessary to check the validity of the weak cosmic censorship conjecture in these cases.

If the event horizon cannot wrap the singularity after the charged particle are absorbed, the weak cosmic censorship conjecture will be invalid. So the event horizon should exist to assure the validity of the weak cosmic censorship conjecture. We will check whether there is an event horizon after the charged particle is absorbed by the black hole. We will pay attention to how $f(r)$ changes. The function $f(r)$ has a minimum point at $r_{\min }$. There are three possibilities. For the case $f(r)_{\min }<0$, there are two roots of $f(r)$. Then we have an usual black hole with $r_{+}$and $r_{-}$, as the inner horizon and outer horizon. For the case $f(r)_{\min }=0$, the two roots coincide, and the black hole becomes into an extremal black hole. For the case $f(r)_{\min }>0$, the function $f(r)$ has no real root, there is not an event horizon. At $r_{\min }$, we have

$$
\left.f(M, Q, l, r)\right|_{r=r_{\min }} \equiv f_{\min }=\delta \leq 0,\left.\quad \partial_{r} f(M, Q, l, r)\right|_{r=r_{\min }} \equiv f_{\min }^{\prime}=0,
$$

and

$$
\left.\left(\partial_{r}\right)^{2} f(M, Q, l, r)\right|_{r=r_{\min }}>0 .
$$

For the extremal black hole, $\delta=0, r_{h}$ and $r_{\min }$ are coincident. For the near-extremal black hole, $\delta$ is a small quantity. When the black hole absorbs charged particle, there are infinitesimal change in the mass, charge and AdS radius, which are $(M+d M, Q+d Q, l+d l)$. Because of these changes, there are also movements for the minimum value and event horizon of the black hole, namely $r_{\min } \rightarrow r_{\min }+d r_{\min }$ and $r_{h} \rightarrow r_{h}+d r_{h}$. At the new minimum point, the function $f(r)$ satisfies

$$
\left.\partial_{r} f(M+d M, Q+d Q, l+d l, r)\right|_{r=r_{\min }+d r_{\min }}=f_{\min }^{\prime}+d f_{\min }^{\prime}=0 .
$$

Using the known condition $f_{\min }^{\prime}=0$, we can obtain $d f_{\min }^{\prime}=0$. Expanding it further, we get

$$
d f_{\min }^{\prime}=\frac{\partial f_{\min }^{\prime}}{\partial M} d M+\frac{\partial f_{\min }^{\prime}}{\partial Q} d Q+\frac{\partial f_{\min }^{\prime}}{\partial l} d l+\frac{\partial f_{\min }^{\prime}}{\partial r_{\min }} d r_{\min }=0 .
$$

At $r_{\min }+d r_{\min }$, the function $f(r)$ takes the form as

$$
\begin{aligned}
f(M+d M, Q+d Q, l+d l, r) & \left.\right|_{r=r_{\min }+d r_{\min }}=f_{\min }+d f_{\min } \\
= & \delta+\left(\frac{\partial f_{\min }}{\partial M} d M+\frac{\partial f_{\min }}{\partial Q} d Q+\frac{\partial f_{\min }}{\partial l} d l\right) .
\end{aligned}
$$

For extremal black hole, we know $f_{\min }^{\prime}=0$ and $f_{\min }=\delta=0$. Substituting equation (25) into equation (37), we get

$$
d f_{\min }=-\frac{2 p^{r}}{r_{\min }}-\frac{3 r_{\min } d r_{\min }}{l^{2}} .
$$

Form equations $(29)$ and $(38)$, we find

$$
d f_{\min }=0 .
$$

For the near-extremal black hole, equation 25 can not be applicable no longer. But we can expand it near the 
minimum point for there is a relation $r_{h}=r_{\min }+\epsilon$. To the first order, we find

$$
\begin{aligned}
d M & =\frac{r_{\min }^{-2-3 \omega} d r_{\min }\left(a l^{3} r_{\min }+3 a l^{3} \omega r_{\min }-2 l^{3} Q^{2} r_{\min }^{3 \omega}+2 l^{3} M r_{\min }^{1+3 \omega}+2 l r_{\min }^{4+3 \omega}\right)}{2 l^{3}} \\
& +\frac{r_{\min }^{-2-3 \omega}\left(2 l^{3} Q r_{\min }^{1+3 \omega} d Q-2 r_{\min }^{5+3 \omega} d l\right)}{2 l^{3}} \\
& -\frac{r_{\min }^{-3-3 \omega} d r_{\min }\left(a l^{3} r_{\min }+6 a l^{3} \omega r_{\min }+9 a l^{3} \omega^{2} r_{\min }\right) \epsilon}{2 l^{3}} \\
& -\frac{r_{\min }^{-3-3 \omega} d r_{\min }\left(-4 l^{3} Q^{2} r_{\min }^{3 \omega}+2 l^{3} M r_{\min }^{1+3 \omega}-4 l r_{\min }^{4+3 \omega}\right) \epsilon}{2 l^{3}} \\
& -\frac{r_{\min }^{-3-3 \omega}\left(2 l^{3} Q r_{\min }^{1+3 \omega} d Q+6 r_{\min }^{5+3 \omega} d l\right) \epsilon}{2 l^{3}}+\mathcal{O}(\epsilon)^{2} .
\end{aligned}
$$

Substituting (40) into (37), we have

$$
\begin{aligned}
d f_{\min } & =\frac{2 Q d Q}{r_{\min }^{2}}-\frac{2 r_{\min }^{2} d l}{l^{3}}-\frac{r_{\min }^{-3-3 \omega}\left(2 l^{3} Q r_{\min }^{1+3 \omega} d Q-2 r_{\min }^{5+3 \omega} d l\right)}{l^{3}} \\
& -\frac{r_{\min }^{-3-3 \omega} d r_{\min }\left(a l^{3} r_{\min }+3 a l^{3} \omega r_{\min }-2 l^{3} Q^{2} r_{\min }^{3 \omega}+2 l^{3} M r_{\min }^{1+3 \omega}+2 l r_{\min }^{4+3 \omega}\right)}{l^{3}} \\
& +\frac{r_{\min }^{-4-3 \omega} d r_{\min }\left(a l^{3} r_{\min }+6 a l^{3} \omega r_{\min }+9 a l^{3} \omega^{2} r_{\min }\right) \epsilon}{l^{3}} \\
& +\frac{r_{\min }^{-4-3 \omega} d r_{\min }\left(-4 l^{3} Q^{2} r_{\min }^{3 \omega}+2 l^{3} M r_{\min }^{1+3 \omega}-4 l r_{\min }^{4+3 \omega}\right) \epsilon}{l^{3}} \\
& +\frac{r_{\min }^{-4-3 \omega}\left(2 l^{3} Q r_{\min }^{1+3 \omega} d Q+6 r_{\min }^{5+3 \omega} d l\right) \epsilon}{l^{3}}+\mathcal{O}(\epsilon)^{2} .
\end{aligned}
$$

For the equation $f\left(r_{h}\right)=0$, we also can expand and solve it, then we find

$$
l=\frac{\sqrt{3} r_{\min }^{\frac{1}{2}(4+3 \omega)}}{\sqrt{-3 a \omega r_{\min }+Q^{2} r_{\min }^{3 \omega}-r_{\min }^{2+3 \omega}}},
$$

Differentiating both sides of this equation, we can get further

$$
\begin{aligned}
d l= & -\frac{\sqrt{3} Q r_{\min }^{3 \omega+\frac{1}{2}(4+3 \omega)} d Q}{\left(-3 a \omega r_{\min }+Q^{2} r_{\min }^{3 \omega}-r_{\min }^{2+3 \omega}\right)^{3 / 2}} \\
& +\frac{\sqrt{3}(4+3 \omega) r_{\min }^{-1+\frac{1}{2}(4+3 \omega)} d r_{\min }}{2 \sqrt{-3 a \omega r_{\min }+Q^{2} r_{\min }^{3 \omega}-r_{\min }^{2+3 \omega}}} \\
& -\frac{\sqrt{3} r_{\min }^{\frac{1}{2}(4+3 \omega)}\left(-3 a \omega+3 Q^{2} \omega r_{\min }^{-1+3 \omega}-(2+3 \omega) r_{\min }^{1+3 \omega}\right) d r_{\min }}{2\left(-3 a \omega r_{\min }+Q^{2} r_{\min }^{3 \omega}-r_{\min }^{2+3 \omega}\right)^{3 / 2}} .
\end{aligned}
$$

Substituting equations (43) and 42 into equation (41), we find

$$
d f_{\min }=\mathcal{O}\left(\epsilon^{2}\right),
$$

then, we can get

$$
f_{\min }+d f_{\min }=\delta+\mathcal{O}\left(\epsilon^{2}\right) .
$$

In Ref. [49], $\delta$ is a very small negative value and $\epsilon \ll 1$. When we chose $\delta=\epsilon=0$, the equation (45) return to the form of the extremal black hole. This result also proves the correctness of the equation (39) from the side. Hence, they think that the extremal and near-extremal black holes stay at their minimum. For the near-extremal black hole, they ignore the $O\left(\epsilon^{2}\right)$ due to it is high order small value, so that they think that the weak cosmic censorship is also valid for the case of the near-extremal black hole. However, because we don't know the magnitude of $|\delta|$ and 
$O\left(\epsilon^{2}\right)$, we can not easily ignore $O\left(\epsilon^{2}\right)$. Therefore, it would be important to find out whether there is a relationship $|\delta|>\mathcal{O}\left(\epsilon^{2}\right)$. For the near-extremal black hole, the minimum point is located slightly to the left of the outer horizon. So the value of the minimum is obtained at $r_{\min }+\epsilon$ as

$$
\begin{aligned}
f\left(r_{\min }+\epsilon\right) & =\left(1-\frac{Q^{2}}{r_{\min }^{2}}+\frac{3 r_{\min }^{2}}{l^{2}}+3 a \omega r_{\min }^{-1-3 \omega}\right) \\
& +\left(\frac{3}{l^{2}}+\frac{1}{2} r_{\min }^{-4-3 \omega}\left(-3 a \omega(1+3 \omega) r_{\min }+2 Q^{2} r_{\min }^{3 \omega}\right)\right) \epsilon^{2}+\mathcal{O}\left(\epsilon^{3}\right),
\end{aligned}
$$

where we skip to write the constant term. Namely

$$
\delta=-\left(\frac{3}{l^{2}}+\frac{1}{2} r_{\min }^{-4-3 \omega}\left(-3 a \omega(1+3 \omega) r_{\min }+2 Q^{2} r_{\min }^{3 \omega}\right)\right) \epsilon^{2}-\mathcal{O}\left(\epsilon^{3}\right) .
$$

And we can also get

$$
d f \min =\left(\frac{\left(8 Q^{2}-2 r_{\min }^{2}\right) d r_{\min }-4 Q r_{\min } d Q}{r_{\min }^{5}}-\frac{27}{2} a \omega(1+\omega)^{2} r_{\min }^{-4-3 \omega} d r_{\min }\right) \epsilon^{2}+\mathcal{O}\left(\epsilon^{3}\right) .
$$

the equation 45 can be represented as

$$
\begin{aligned}
f\left(r_{\min }+d r_{\min }\right) & =\left(\frac{\left(8 Q^{2}-2 r_{\min }^{2}\right) d r_{\min }-4 Q r_{\min } d Q}{r_{\min }^{5}}-\frac{27}{2} a \omega(1+\omega)^{2} r_{\min }^{-4-3 \omega} d r_{\min }\right) \epsilon^{2} \\
& -\left(\frac{3}{l^{2}}+\frac{1}{2} r_{\min }^{-4-3 \omega}\left(-3 a \omega(1+3 \omega) r_{\min }+2 Q^{2} r_{\min }^{3 \omega}\right)\right) \epsilon^{2} .
\end{aligned}
$$

Here, we can have $\mathcal{F}=\frac{f\left(r_{\min }+\mathrm{dr}_{\min }\right)}{\epsilon^{2}}$ and $e=d Q$. Then, the above equation becomes

$$
\begin{aligned}
\mathcal{F}= & \frac{\left(8 Q^{2}-2 r_{\min }^{2}\right) d r_{\min }-4 Q r_{\min } e}{r_{\min }^{5}}-\frac{27}{2} a \omega(1+\omega)^{2} r_{\min }^{-4-3 \omega} d r_{\min } \\
& -\frac{1}{2} r_{\min }^{-4-3 \omega}\left(-3 a \omega(1+3 \omega) r_{\min }+2 Q^{2} r_{\min }^{3 \omega}\right)-\frac{3}{l^{2}},
\end{aligned}
$$

obviously, from above equation we find that the values of $\mathcal{F}$ is link with the values of the parameters $e, a, l, r_{\min }, \omega, Q, d r_{\min }$. Mostly, we find that $e$ has a more direct affect on $\mathcal{F}$. To gain an intuitive understanding, we plot Figure 3 in follow
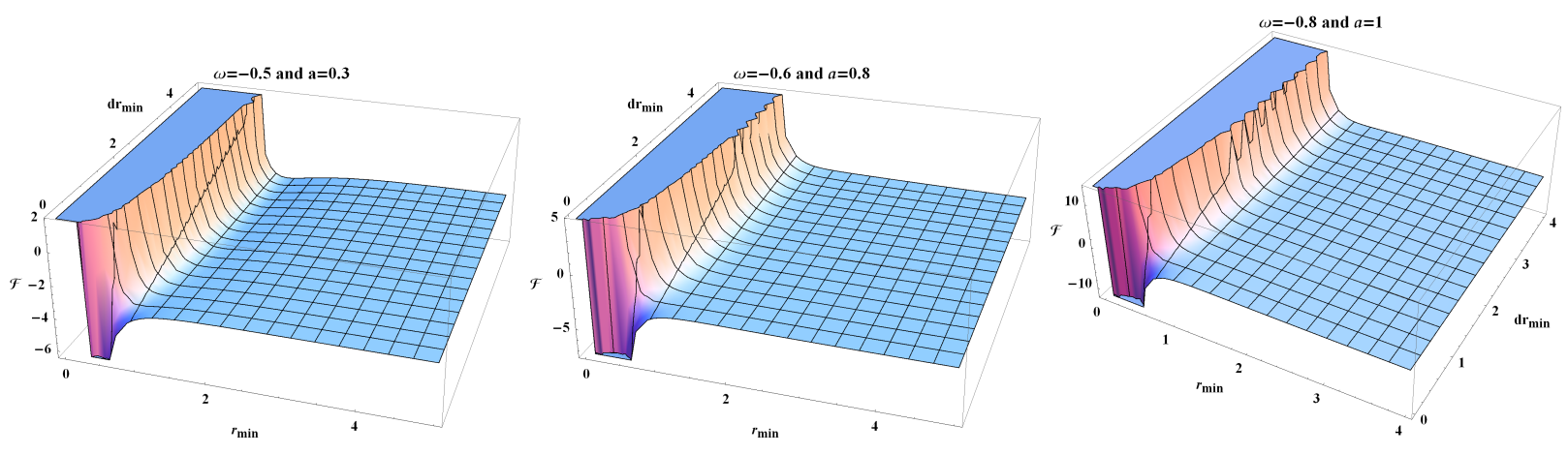

Fig. 3. The value of $\mathcal{F}$ for $l=1, Q=0.5, e=0.2$.

When we take different values of these parameters, the region where $\mathcal{F}$ is greater than zero always appears in the graph. $\mathcal{F}>0$, that is, $f\left(r_{\min }+d r_{\min }\right)>0$. In other words, the positive region shows that there exists a rang of the particle charge which allow us to overcharge black holes into naked singularities. Similarly, for different values of the parameters $a, l, r_{\min }, \omega, Q, d r_{\min }$, the configurations of $\mathcal{F}$ are different, that is, the violation about the weak cosmic censorship depend on the parameters and the magnitudes of the violation is related to those of the parameters. Especially, when we take $e<<1$, and show in the Figure 4, we still find the same violation. However, the degree of violation is different as the change of the charge $e$ 


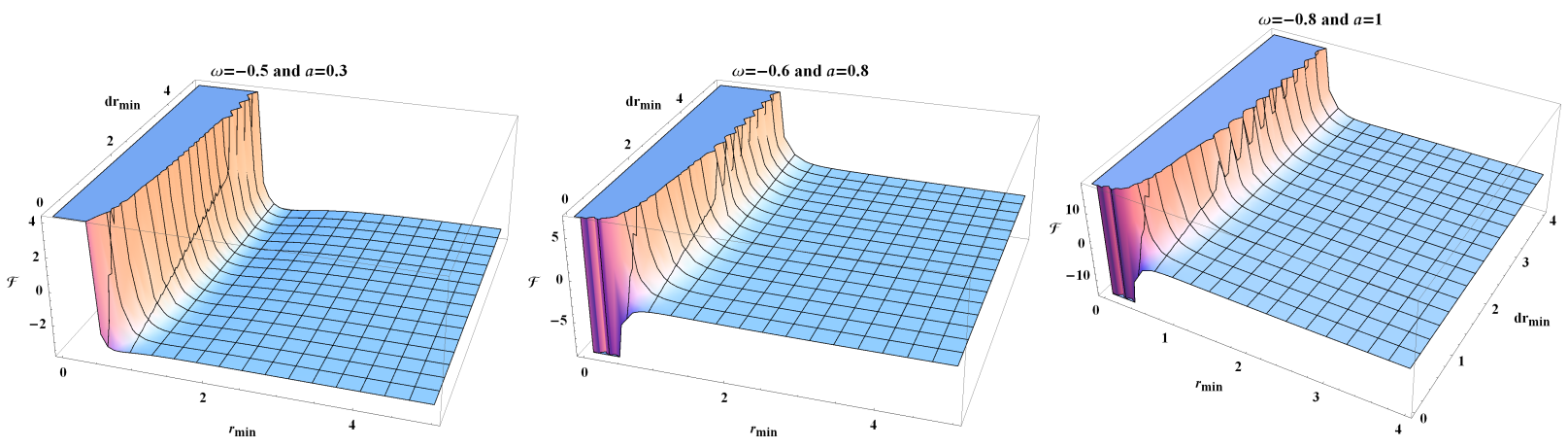

Fig. 4. The value of $\mathcal{F}$ for $l=1, Q=0.5, e=0.00005$.

In the extended phase space, we find that the change of the values of $f\left(r_{\min }\right)$ vanishes always after the charged particle is absorbed for the extremal black hole. That is, for the extremal black holes, the function $f(r)$ always has a root. Therefore, the black hole has an event horizon covering its singularity. The weak cosmic censorship conjecture thus is valid for the configuration of the black hole does not change. However, we derive that the near-extremal black holes can be overcharged by absorbing charged particle. Hence, the cosmic censorship conjecture would be violated for the near-extremal black holes in the extended phase space.

When $a$ is zero, the black hole becomes RN-AdS black holes. Hence, we set $a=0$, and the result is shown in Figure 5 .
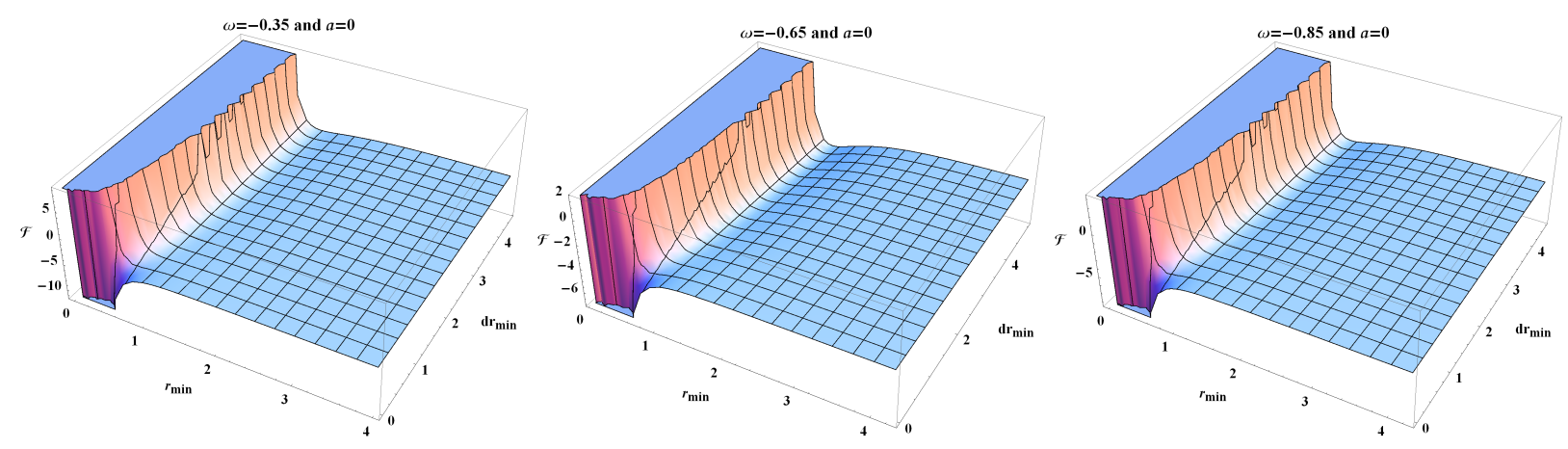

Fig. 5. The value of $\mathcal{F}$ for $l=1, Q=0.5, e=0.2$.

Interestingly, the results show that not only $\mathcal{F}<0$ but also $\mathcal{F}>0$, which means the weak cosmic censorship may be invalid for the RN-AdS black holes, and the results are some different with the previous works [49] about the weak cosmic censorship. The reasons is that they did not consider the high order corrections to the energy.

\section{Thermodynamics and the weak cosmic censorship conjecture without contributions of pressure and volume}

In this section, we mainly discuss the laws of thermodynamics and examine the weak cosmic censorship conjecture of the black hole in the normal phase space. We want to explore whether the phase space affects thermodynamics and weak cosmic censorship conjecture.

\subsection{Thermodynamic in the normal phase space}

In the normal phase space, the mass $M$ of the black hole is defined as energy. Meanwhile, we assume that there is no energy loss in the process of particle absorption. As the charged particle is absorbed by the black hole, the change of the internal energy and charge of the black hole satisfy

$$
E=d M, \quad e=d Q .
$$


Then, equation (19) can be rewritten as

$$
d M=\frac{Q}{r_{h}} d Q+p^{r} .
$$

Due to the absorption of a charged particle, the event horizon and function $f\left(r_{h}\right)$ will change, and there is always a relation

$$
d f_{h}=\frac{\partial f_{h}}{\partial M} d M+\frac{\partial f_{h}}{\partial Q} d Q+\frac{\partial f_{h}}{\partial r_{h}} d r_{h}=0 .
$$

In order to eliminate the $d M$ term, we can combine equations 52 and 53 . We find that the $d Q$ term is also eliminated in this process and we get lastly

$$
d r_{h}=\frac{2 l^{2} p^{r} r_{h}{ }^{2}}{2 l^{2} r_{h}{ }^{2}-2 M l^{2} r_{h}+4 r_{h}{ }^{4}+a l^{2} r_{h}{ }^{-(3 \omega-1)}(3 \omega-1)} .
$$

After substituting equation (54) into equation (22), we have

$$
d S_{h}=\frac{4 \pi l^{2} p^{r} r_{h}^{3}}{2 l^{2} r_{h}{ }^{2}-2 M l^{2} r_{h}+4 r_{h}{ }^{4}+a l^{2} r_{h}{ }^{-(3 \omega-1)}(3 \omega-1)} .
$$

Form equations (4) and (55), we get

$$
T_{h} d S_{h}=p^{r}
$$

Combining equations (4), (6) and (55), we get

$$
d M=T_{h} d S_{h}+\Phi_{h} d Q .
$$

In the normal phase space, the first law of thermodynamics of the black hole surrounded by quintessence dark energy is valid when a particle is absorbed by the black hole.

With equations (55), we also can investigate the second law of thermodynamics. For the extremal black holes, we find the variation of the entropy is divergent, which is meaningless. So we are interested in the near-extremal black hole thereafter. We also set $M=0.5$ and $l=p^{r}=1$. For case of $\omega=-1 / 2$ and $a=1 / 2$, we get the extremal charge $Q_{e}=0.525694072$. In the case that the charge is less than the extreme charge, we obtain values of $r_{h}$ and $d S_{h}$ for different charge in Table 3. From this table, it can be clearly seen that when the charge is smaller than the extremal charge, the variation of the entropy is positive always. In Figure 6, we present the relation between $d S_{h}$ and $r_{h}$, we can see that the entropy increases too. Therefore, the second law of thermodynamics is valid in the normal phase space.

Table 3. The relation between $\mathrm{dS}_{h}, Q$ and $r_{h}$.

\begin{tabular}{ccc}
\hline$Q$ & $r_{h}$ & $d S_{h}$ \\
\hline 0.525694072 & 0.483844 & 39998.1 \\
0.525 & 0.504022 & 43.7772 \\
0.5 & 0.599642 & 9.3879 \\
0.4 & 0.713732 & 5.67468 \\
0.3 & 0.767156 & 4.92923 \\
0.2 & 0.798062 & 4.60478 \\
0.1 & 0.814681 & 4.45287
\end{tabular}

For the case $\omega=-2 / 3, a=1 / 3$, we find the extremal charge is $Q_{e}=0.48725900875$. the values of $r_{h}$ and $d S_{h}$ for different charge are given in Figure 7 and Table 4 From them, we also find that the entropy increase, implying that the second law is valid.

Table 4. The relation between $d S_{h}, Q$ and $r_{h}$.

\begin{tabular}{ccc}
\hline$Q$ & $r_{h}$ & $d S_{h}$ \\
\hline 0.48725900875 & 0.432041 & $3.47995 \times 10^{6}$ \\
0.487259 & 0.434211 & 322.509 \\
0.48725 & 0.434319 & 307.346 \\
0.4 & 0.628743 & 5.80081 \\
0.3 & 0.695450 & 4.85183 \\
0.2 & 0.731837 & 4.49911 \\
0.1 & 0.751055 & 4.34168 \\
\hline
\end{tabular}




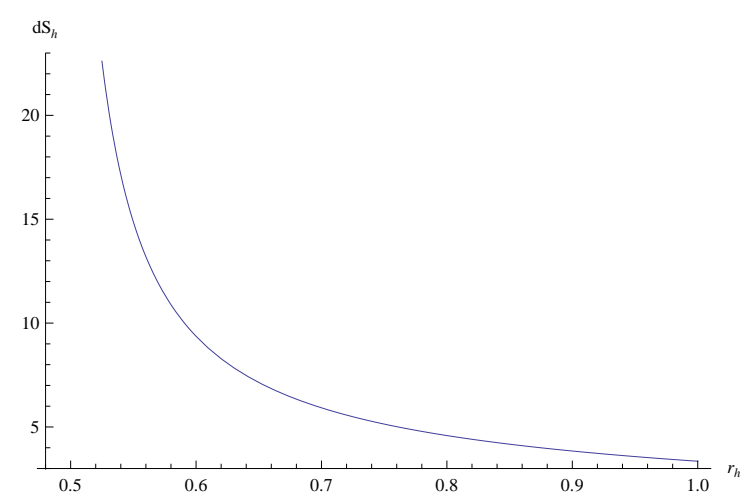

Fig. 6. The relation between $d S_{h}$ and $r_{h}$ which parameter values are $a=1, M=0.5, l=p^{r}=1$.

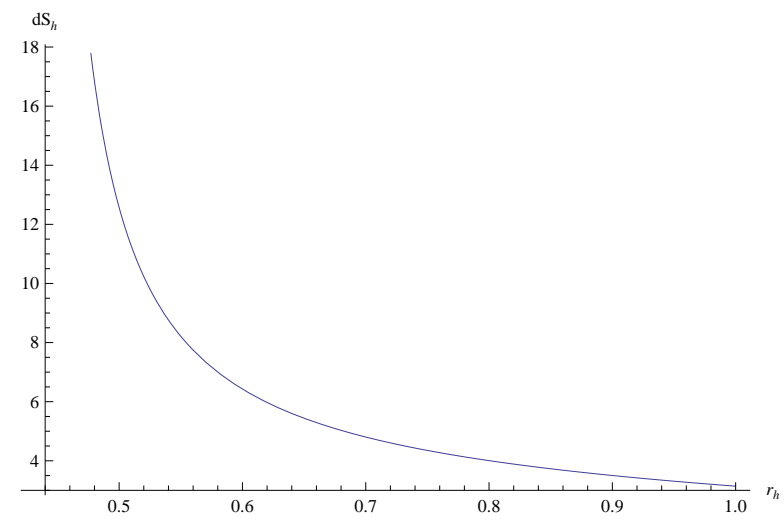

Fig. 7. The relation between $d S_{h}$ and $r_{h}$ which parameter values are $a=1 / 3, M=0.5, l=p^{r}=1$.

Thus far, both the first and second laws of thermodynamics hold in the normal phase space for the black hole surrounded by quintessence dark energy under charged particle absorption.

\subsection{Weak Cosmic Censorship Conjecture in the normal phase space}

In the normal phase, the examination of the validity of the weak cosmic censorship conjecture should also return to the value of the function $f\left(r_{\min }\right)$. Similarly, we will study how $f\left(r_{\min }\right)$ changes as charged particle are absorbed. At $r_{\min }+d r_{\min }$, there is also a relation $\partial_{r} f\left(r_{\min }+d r_{\min }\right)=0$, implying

$$
d f_{\min }^{\prime}=\frac{\partial f_{\min }^{\prime}}{\partial M} d M+\frac{\partial f_{\min }^{\prime}}{\partial Q} d Q+\frac{\partial f_{\min }^{\prime}}{\partial r_{\min }} d r_{\min }=0
$$

In addition, at the new minimum point, $f\left(r_{\min }+d r_{\min }\right)$ can be expressed as

$$
f\left(r_{\min }+d r_{\min }\right)=f_{\min }+d f_{\min },
$$

where

$$
d f_{\min }=\frac{\partial f_{\min }}{\partial M} d M+\frac{\partial f_{\min }}{\partial Q} d Q .
$$

For the extremal black holes, equation (52) can be applied. In this case, we have $f_{\min }=\delta=0$, inserting equation (52) into equation 60), we can finally get

$$
d f_{\min }=-\frac{2 p^{r}}{r_{\min }} .
$$

When it is an extreme black hole, $r_{h}$ and $r_{\min }$ are tightly coincident. In addition, we have $T_{h}=0$. Incorporating equation (61) and equation (56), the minimum value of $f\left(r_{\min }+d r_{\min }\right)$ becomes

$$
f\left(r_{\min }+d r_{\min }\right)=0,
$$


which shows that $f_{\min }+d f_{\min }=0$, so that the charged particle does not change the minimum value. Therefore, the weak cosmic censorship conjecture is valid for the extremal black hole surrounded by quintessence dark energy. It is interesting to note that this result same with that in the extended phase space, the configuration of the black hole has not change after the absorption, the extremal black hole is still an extremal black hole.

For the near-extremal black hole, equation (52) can not be used. With the condition $r_{h}=r_{\min }+\epsilon$, we can expand equation 52 at $r_{\min }$, which leads to

$$
\begin{aligned}
d M & =\frac{Q}{r_{\min }} d Q \\
& +\left(Q^{2} d r_{\min }+\frac{3}{2} r_{\min }\left(\frac{2 r_{\min }{ }^{3}}{l^{2}}-a r_{\min }{ }^{-3 \omega} \omega(1+3 \omega)\right) d r_{\min }-Q r_{\min } d Q\right) r_{\min }{ }^{-3} \epsilon \\
& +\frac{1}{4} r_{\min }{ }^{-4-3 \omega}\left(4 Q r_{\min }{ }^{1+3 \omega} d Q+d r_{\min }\left(-8 Q^{2} r_{\min }{ }^{3 \omega}+9 a r_{\min } \omega\left(1+4 \omega+3 \omega^{2}\right)\right)\right) \epsilon^{2} \\
& +\mathcal{O}\left(\epsilon^{3}\right) .
\end{aligned}
$$

By combining equations 60 and 63 we have

$$
d f_{\min }=\frac{1}{2} r_{\min }^{-4-3 \omega}\left(4 r_{\min }^{1+3 \omega}-9 a \omega\left(2 \omega+3 \omega^{2}-1\right)\right) \epsilon^{2} d r_{\min }+\mathcal{O}\left(\epsilon^{3}\right) .
$$

We do the same calculation that we did in the extended phase space. For the near-extremal black hole, we also can define $\mathcal{F}_{N}=\frac{f\left(r_{\min }+\mathrm{dr}_{\min }\right)}{\epsilon^{2}}$. Hence, we can get the express of $\mathcal{F}_{N}$ in the normal phase as

$$
\mathcal{F}_{N}=\frac{4 r_{\min } d r_{\min }-2 Q^{2}+3 a r_{\min }{ }^{-3 w} \omega\left(r_{\min }+3 r_{\min } \omega-3 d r_{\min }(1+\omega)(3 \omega-1)\right)}{2 r_{\min }{ }^{4}}-\frac{3}{l^{2}},
$$

From the equation (65), it is not easily determine the value of $\mathcal{F}_{N}$ is positive or negative. Similar with the extended phase space, we still have Figure 8 and Figure 9.

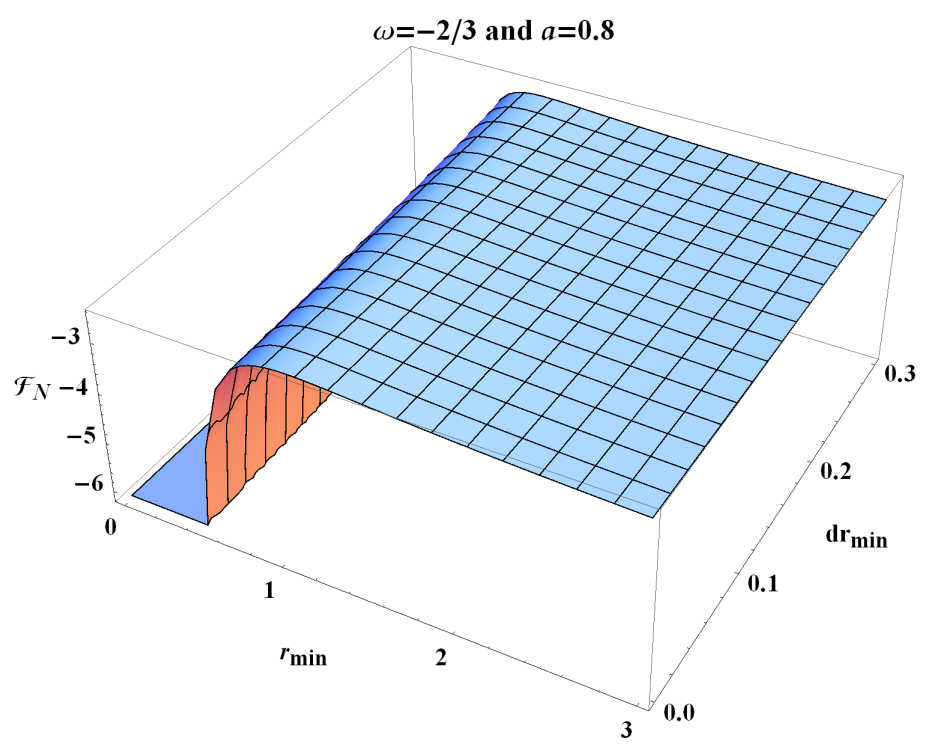

Fig. 8. The value of $\mathcal{F}_{N}$ for $Q=0.5, l=a=1$ and $\omega=-0.5$.

In Figure 8 and Figure 9, there is no region where the value of $\mathcal{F}_{N}$ is positive. Concerning this result, it is important to note that the conclusion is very different from the extended phase space. In other word, we always have $f\left(r_{\text {min }}+d r_{\text {min }}\right)<0$ for the near-extremal black hole in the normal phase space. Therefore, the weak cosmic censorship conjecture of the near-extremal black hole surrounded by quintessence dark energy is valid under charged particle absorption in the normal phase space. 


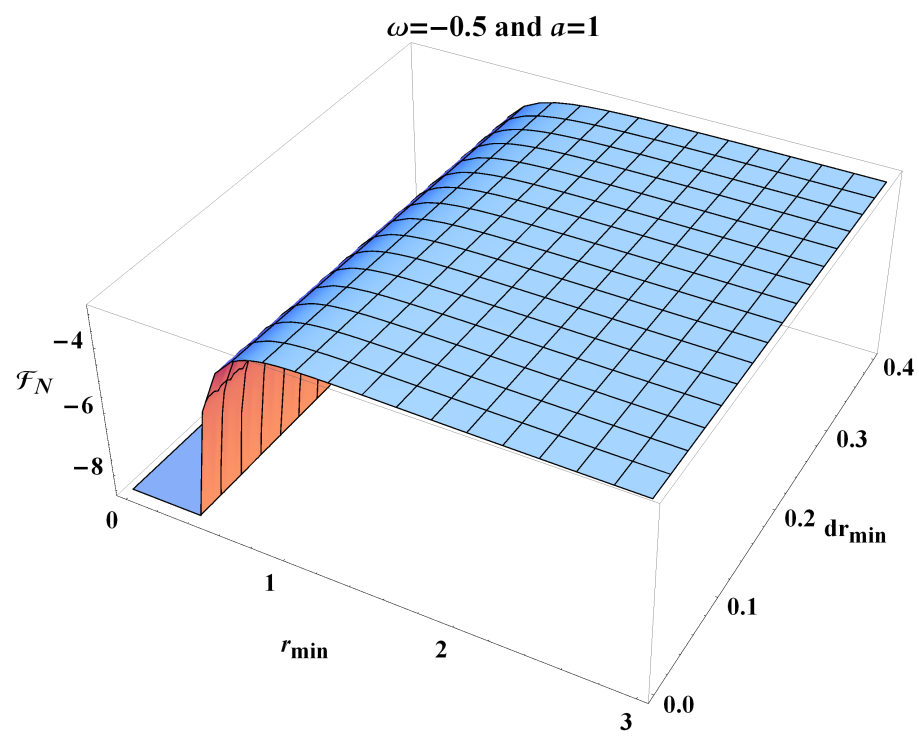

Fig. 9. The value of $\mathcal{F}_{N}$ for $Q=0.5, l=a=1$ and $\omega=-2 / 3$.

\section{Discussion and conclusions}

In the extended phase space, the cosmological parameter has been set to be a dynamic variable and interpreted as the pressure. When the charged particle is absorbed by the black hole surrounded with quintessence dark energy, the change of the energy and the charge of the black hole were supposed to be equal to the conserved quantity of the charged particle. In this case, we found that the first law of thermodynamics was completely valid, but the second law of thermodynamics was violated for extremal and near-extremal black holes. We also studied the validity of the weak cosmic censorship conjecture. We mainly calculated the change of the minimum value of the function $f(M, Q, l, r)$ under the charged particle absorption by studying the minimum value of the function $f(M, Q, l, r)$. Firstly, we calculated the case of the extremal black holes. We found that the minimum value of the function was not changed under the charged particle absorption. In other words, after the charge particle absorption, the extremal black hole stays extremal. Therefore, the weak cosmic censorship conjecture is valid for the extremal black hole in the extended phase space. However, our results shown that the minimum value of the function under the absorption would appear the case of greater than zero. It has shown that the near-extremal black hole could be overcharged, which was different from the extremal black hole. That is, the cosmic censorship conjecture would be violated for the near-extremal black hole surrounded by quintessence dark energy after absorbs the charged particle in the extended phase space.

In this paper, the results for the violation of the weak cosmic censorship conjecture in the extended phase space are dissimilar from previous conclusions. In previous research [49, they regarded the second order $\mathcal{O}\left(\epsilon^{2}\right)$ of $d f_{\min }$ as a very small quantity, so that they neglected the contribution of $\mathcal{O}\left(\epsilon^{2}\right)$ to $d f_{\min }$. Here, we made precise comparisons and calculations at the minimum value of the function, and we obtained the magnitudes relationship between $\delta$ and $\mathcal{O}\left(\epsilon^{2}\right)$. We found there was always a case of $f\left(r_{\min }+d r_{\min }\right)>0$, which means that the weak cosmic censorship conjecture may be violated. And we still observes such violations under assuming $e<<1$. Interestingly, when we taken $a=0$, the black hole returns to the RN-AdS black hole, and the cosmic censorship conjecture was also invalid, which is different from the result in 49 .

In normal phase space, the cosmological constant is definite. We found that the first and second laws of thermodynamics are both satisfied under the particle absorption. In addition, the weak cosmic censorship conjecture was also checked. For the case of extremal black hole, we found that the configuration of the black hole surrounded by quintessence dark energy also has not changed after absorbs charged particle, which is same as in the extended phase space. The result implies that the extremal black hole could not be overcharged in the normal phase space. Interestingly, for the case of near-extremal black hole, the minimum value of the function is still negative when the particle is absorbs into the black hole, which is different form that in the extended phase space. That is, the weak cosmic censorship conjecture is valid for the near-extremal black holes in the normal phase space. 


\section{Acknowledgements}

This work is supported by the National Natural Science Foundation of China (Grant Nos. 11875095), and Basic Research Project of Science and Technology Committee of Chongqing (Grant No. cstc2018jcyjA2480).

\section{References}

1 R. Penrose, Phys. Rev. Lett. 14, 57 (1965).

2 R. Penrose, Riv. Nuovo Cim. 1, 252 (1969).

3 S. W. Hawking and R. Penrose, Proc. Roy. Soc. Lond. A 314, 529 (1970).

4 R. M. Wald, Annals. Phys. 82, 548 (1974).

5 I. Semiz, Gen. Relat. Grav. 43, 833 (2010).

6 G. Z. Toth, Gen. Relativ. Gravit. 44, 2019 (2012).

7 V. E. Hubeny, Phys. Rev. D 59, 064013 (1999).

8 T. Jacobson and T. P. Sotiriou, Phys. Rev. Lett. 103, 141101 (2009).

9 A. Saa, R. Santarelli, Phys. Rev. D 84, 027501 (2011).

10 E. Barausse, V. Cardoso and G. Khanna, Phys. Rev. Lett. 105, 261102 (2010).

11 E. Barausse, V. Cardoso and G. Khanna, Phys. Rev. D 84, 104006 (2011).

12 M. Colleoni, L. Barack, A. G. Shah and M. van de Meent, Phys. Rev. D 92, no. 8, 084044 (2015).

13 V. E. Hubeny, Phys. Rev. D 59, 064013 (1999).

14 S. Isoyama, N. Sago and T. Tanaka, Phys. Rev. D 84, 124024 (2011).

15 M. Colleoni and L. Barack, Phys. Rev. D 91, 104024 (2015).

16 M. Bouhmadi-Lopez, V. Cardoso, A. Nerozzi and J. V. Rocha, Phys. Rev. D 81, 084051 (2010).

17 S. Hod, Class. Quant. Grav. 33, no. 3, 037001 (2016).

18 G. T. Horowitz, J. E. Santos and B. Way, Class. Quant. Grav. 33, no. 19, 195007 (2016).

19 X. X. Zeng, J. S. Hou and S. Z. Yang, Pramana 70, 409 (2008).

20 Y. P. Hu, X. X. Zeng and H. Q. Zhang, Phys. Lett. B 765, 120 (2017).

21 K. Düztaş, Phys. Rev. D 94, no. 12, 124031 (2016).

22 J. Natario, L. Queimada and R. Vicente, Class. Quant. Grav. 33, no. 17, 175002 (2016).

23 S. Gao and Y. Zhang, Phys. Rev. D 87, no. 4, 044028 (2013).

24 J. V. Rocha, R. Santarelli and T. Delsate, Phys. Rev. D 89, no. 10, 104006 (2014).

25 J. V. Rocha and R. Santarelli, Phys. Rev. D 89, no. 6, 064065 (2014).

26 J. Sorce and R. M. Wald, Phys. Rev. D 96, no. 10, 104014 (2017).

27 J. V. Rocha and V. Cardoso, Phys. Rev. D 83, 104037 (2011).

28 B. Gwak, RINP 13, 102155 (2019).

29 D. Chen, arXiv:1812.03459 [gr-qc].

30 B. Gwak and B. H. Lee, JCAP 1602, 015 (2016)

31 Y. W. Han, X. X. Zeng, and Y. Hong, arXiv:1901.10660 [gr-qc].

32 X. X. Zeng, Y. W. Han, and D. Chen, Chin. Phys. C 43, no. 10, 105104 (2019).

33 X. X. Zeng and H. Q. Zhang, arXiv:1901.04247[ [hep-th].

34 D. Chen, arXiv:1902.06489 [hep-th].

35 B. Gwak, Phys. Rev. D 95, no. 12, 124050 (2017).

36 X. X. Zeng, X. Y. Hu, K. J. He, arXiv:1905.07750 [hep-th].

37 W. Hong, B. Mu, J. Tao, arXiv:1905.07747 [gr-qc].

38 P. Wang, H. Wu and H. Yang, arXiv:1904.12365 [gr-qc].

39 X. X. Zeng and H. Q. Zhang, arXiv:1905.01618 [gr-qc]].

40 X. X. Zeng and X. Y. Hu, arXiv:1908.03845 [gr-qc].

41 C. Teitelboim, Phys. Lett. 158B, 293 (1985).

42 J. D. Brown and C. Teitelboim, Nucl. Phys. B 297, 787 (1988).

43 M. M. Caldarelli, G. Cognola and D. Klemm, Class. Quant. Grav. 17, 399 (2000).

44 T. Padmanabhan, Class. Quant. Grav. 19, 5387 (2002).

45 B. P. Dolan, Class. Quant. Grav. 28, 125020 (2011).

46 M. Cvetic, G. W. Gibbons, D. Kubiznak and C. N. Pope, Phys. Rev. D 84, 024037 (2011).

47 D. Kastor, S. Ray and J. Traschen, Class. Quant. Grav. 26, 195011 (2009).

48 B. P. Dolan, Class. Quant. Grav. 28, 235017 (2011).

49 B. Gwak, JHEP 1711, 129 (2017)

50 V. V. Kiselev, Class. Quant. Grav. 20, 1187 (2003).

51 X. X. Zeng and L. F. Li, Phys. Lett. B 764, 100 (2017).

52 B. P. Dolan, arXiv:1209.1272 [gr-qc].

53 B. P. Dolan, D. Kastor, D. Kubiznak, R. B. Mann and J. Traschen, Phys. Rev. D 87, no. 10, 104017 (2013).

54 D. Christodoulou, Phys. Rev. Lett. 25, 1596 (1970).

55 R. M. Wald, Int. J. Mod. Phys. D 27, 1843003 (2018). 